\title{
$\mathrm{J} / \psi$ photoproduction in $\mathbf{P b}-\mathbf{P b}$ ultra-peripheral collisions at $\sqrt{\mathrm{s}_{\mathrm{NN}}}=2.76 \mathrm{TeV}$ with ALICE at LHC
}

\section{De Gruttola*}

Centro Fermi Roma and Salerno INFN, Italy

E-mail: daniele.de.gruttola@cern.ch

The photoproduction of vector mesons in Ultra-Peripheral Collisions (UPC) is a powerful tool to probe the nuclear gluon distribution, for which there is considerable uncertainty in the low- $x$ region. We present the first measurements in $\mathrm{Pb}-\mathrm{Pb}$ collisions at $\sqrt{\mathrm{s}_{\mathrm{NN}}}=2.76 \mathrm{TeV}$, performed with the ALICE detector. The $\mathrm{J} / \psi$ is identified via its dimuon decay in the forward rapidity region and via dimuon and dielectron decay at midrapidity. The results are compared to theoretical models for coherent $\mathbf{J} / \psi$ production and found to be in good agreement with models which include nuclear gluon shadowing. Finally, we present the cross section measurement for incoherent $\mathrm{J} / \psi$.

Photon 2013,

20-24 May 2013

Paris, France

${ }^{*}$ Speaker. 


\section{Ultra-peripheral collisions}

Ultra-peripheral collisions may occur when two accelerated nuclei are separated by impact parameters larger than the sum of their radii; hadronic interactions are strongly suppressed so that both pure electromagnetic and photonuclear processes can be studied. These interactions can be studied in $\mathrm{Pb}-\mathrm{Pb}$ collisions at the LHC at unprecedentedly high energies, where the available centre of mass energy per nucleon pair is $2.76 \mathrm{TeV}$, allowing $\gamma \gamma$ interactions at $\sim 80 \mathrm{GeV}$ and $\gamma p$ at $\sim 480$ $\mathrm{GeV}$, to be compared with 10 and $30 \mathrm{GeV}$ reached at RHIC, respectively. An accelerated charge can be considered as a source of quasi-real photons with $\mathrm{Q}^{2}$ depending on the radius of the nucleus, according to the proposal by Fermi [1] and the relativistic version of Weizsäcker [2] and Williams [3]. One of these quasi-real photons may collide either with the opposite ion or with one of its quasi-real photons. The cross section for photon induced reactions is large because the strong electromagnetic field of the nucleus enhances the intensity of the quasi-real photon flux, which grows as $Z^{2}$, where $Z$ is the charge of the nucleus. The virtuality of the photons is restricted by the nuclear form factor to be of the order $1 / \mathrm{R} \sim 30 \mathrm{MeV} / c$ ( $\mathrm{R}$ is the radius of the nucleus). UPCs lead to the photoproduction of vector mesons $\left(\rho^{0}, \mathrm{~J} / \psi, \psi^{\prime}, \Upsilon, \ldots\right)$ and pure QED processes giving rise to a di-lepton continuum. The physics of ultra-peripheral collisions is reviewed in $[4,5]$.

\section{Physics motivations}

Exclusive photoproduction of vector mesons, where a vector meson but no other particles are produced in the event, is of particular interest. Exclusive production of $\mathrm{J} / \psi$ in photon-proton interactions, $\gamma+\mathrm{p} \rightarrow \mathrm{J} / \psi+\mathrm{p}$, has been successfully modeled in perturbative QCD in terms of the exchange of two gluons with no net-colour transfer [6]. Experimental data on this process from HERA have been used to constrain the proton gluon-distribution at low Bjorken- $x$ [7]. Exclusive vector meson production in heavy-ion interactions is expected to probe the nuclear gluon distribution [8], for which there is considerable uncertainty in the low- $x$ region [9]. A J/ $\psi$ produced at rapidity $y$ is sensitive to the gluon distribution at $\mathrm{x}_{1,2}=\left(\mathrm{M}_{\mathrm{J} / \psi} / \sqrt{\mathrm{S}_{\mathrm{NN}}}\right) \mathrm{e}^{ \pm y}$, where $\mathrm{M}$ is the mass of the vector meson. As an example, at the forward rapidities considered here, $-3.6<y<-2.6$, the relevant values of $x$ are $x_{1} \sim 10^{-2}$ and $x_{2} \sim 10^{-5}$. Exclusive photoproduction can be either coherent, where the photon couples coherently to all nucleons, or incoherent, where the photon couples to a single nucleon. Coherent production is characterized by low vector meson transverse momentum $\left(\left\langle p_{T}\right\rangle \sim 60\right.$ $\mathrm{MeV} / c$ ) and the target nucleus normally does not break up. Incoherent production, corresponding to quasi-elastic scattering off a single nucleon, is characterized by a higher transverse momentum $\left(\left\langle p_{T}\right\rangle \sim 500 \mathrm{MeV} / c\right)$ and the target nucleus normally breaks up; except for single nucleons or nuclear fragments in the very forward region no other particles are produced. In what follows, results on coherent $\mathrm{J} / \psi$ production in the forward region reconstructed in the dimuon decay channel and coherent $\mathrm{J} / \psi$ production at central rapidities in both leptonic decays are presented.

\section{Results and comparison with models}

The ALICE detector [10] allows for a study of UPCs in different rapidity ranges. The ALICE sub-detectors used in this analysis can be found in [10], while [12] describes the trigger and data selection. 


\subsection{Forward rapidity region}

The results for the forward rapidity region have been recently published in [11]. The invariant mass distribution for the selected events and the transverse momentum distribution for events in the mass range $(2.8,3.4) \mathrm{GeV} / \mathrm{c}^{2}$ are shown in Fig. 1. The peak at the $\mathrm{J} / \psi$ mass is clearly seen and has been fitted to an exponential plus a Crystal Ball parameterization. The transverse momentum distribution of the $\mathrm{J} / \psi$ candidates are well reproduced by simulation templates, which take into account the contributions from coherent and incoherent $\mathrm{J} / \psi$ production, the feed-down from $\psi^{\prime}$ and the contribution from the di-muon continuum.
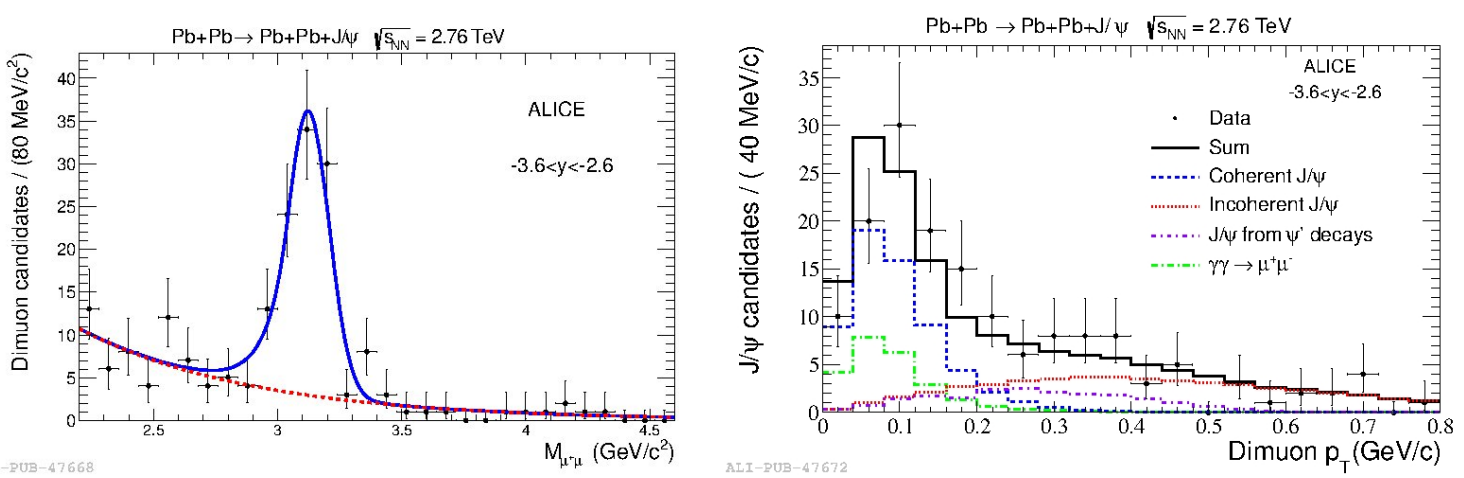

Figure 1: Invariant mass spectrum of forward coherent $\mathrm{J} / \psi \rightarrow \mu^{+} \mu^{-}$candidates with $\mathrm{p}_{T}<300 \mathrm{MeV} / c$ and less than 6 neutrons in the ZDC (left) and $\mathrm{J} / \psi \mathrm{p}_{\mathrm{T}}$ distribution (right) fitted to a simulation template as described in the text.

As expected, at low transverse momentum the coherent production dominates. The yield of coherent $\mathrm{J} / \psi$, obtained by a cut on di-muon momentum below $300 \mathrm{MeV} / \mathrm{c}$ and a ZDC cut tuned to select events with less than 6 emitted neutrons, is estimated to be $\mathrm{N}_{\mathrm{J} / \psi}^{\mathrm{coh}}=78 \pm 10$ (stat) ${ }_{-11}^{+7}$ (syst). The coherent differential cross section at $\sqrt{\mathrm{S}_{\mathrm{NN}}}=2.76 \mathrm{TeV}$ in the rapidity interval $-3.6<y<-2.6$ is given by: $\frac{d \sigma_{J / \psi}^{c o h}}{d y}=\frac{1}{B . R .\left(J / \psi \rightarrow \mu^{+} \mu^{-}\right)} \frac{N_{J / \psi}^{c o h}}{N_{\gamma \gamma}} \frac{(A c c \times \varepsilon)_{\gamma \gamma}}{(A c c \times \varepsilon)_{J / \psi}} \frac{\sigma_{\gamma \gamma}}{\Delta y}$

where B.R. is the branching ratio in the muon channel, $($ Acc $\times \varepsilon)$ is the correction for efficiency and acceptance of the coherent and continuum samples, $\Delta y$ is the width of the rapidity interval and $\mathrm{N}_{\gamma \gamma}$ is the number of $\gamma \gamma$ events in the two mass regions neighbouring the $\mathrm{J} / \psi$ peak; $\sigma_{\gamma \gamma}$ is the cross section for the continuum production, derived from a pure QED calculation. This cross section is known with a precision of about $20 \%$, representing the largest source of systematic errors. In fact the QED coupling, enhanced by $\mathrm{Z}$ (the charge of the ion), is not perturbatively small and higher-order corrections are potentially large; there are uncertainties on the minimum momentum transfer and the nuclear form factor.

The resulting cross section is $d \sigma_{J / \psi}^{c o h} / d y=1.00 \pm 0.18$ (stat) ${ }_{-0.26}^{+0.24}$ (syst) mb.

\subsection{Mid-rapidity region}

The invariant mass distribution is shown in Fig. 2, separated in the coherent (top plots), incoherent samples (bottom plots) and decay channels to dimuons (right) and dielectron (left). Data are fitted with an exponential curve and a Crystal Ball function. In the muon channel the coherence 
condition is applied by requiring a transverse momentum lower than $200 \mathrm{MeV} / c$. The enriched coherent sample, besides the $\mathrm{J} / \psi$ peak, shows also a $\psi$ ' peak arising above the dimuon continuum (top panels). The contribution from the like-sign pairs (negligible in the dielectron channel) is present in the incoherent enriched sample for the dimuon channel (bottom, right) and it is likely to come from misidentified $\pi^{+} \pi^{-}$pairs; it was taken into account by using a polynomial function in the corresponding fit. In the electron channel the effects of the bremsstrahlung due to the detector material is visible in the width and asymmetry of the signal and the coherence conditions is better fulfilled with a $p_{T}$ cut below $300 \mathrm{MeV} / c$. In both cases less than 6 neutrons in the Zero Degree Calorimeter (ZDC) are required to get the coherence condition. The transverse momentum distribution of dielectrons and dimuons can be found in [12]. The templates reproduce well the data when one takes into account the contributions from coherent and incoherent $\mathrm{J} / \psi$ production, the feed-down from coherent and incoherent $\psi^{\prime}$, the contribution of dilepton continuum and finally a contribution from $\mathrm{J} / \psi$ produced in very peripheral hadronic interactions.

The cross section is $d \sigma_{J / \psi}^{c o h} / d y=2.38_{-0.24}^{+0.34}$ (stat+syst) mb and the results have been recently published in [12].

\subsection{Comparison with theoretical calculations}

The results at forward and central rapidities are compared to theoretical predictions in Fig. 3. Theoretical predictions are presented in this plot. The description of all these models can be found in [12].

\section{Summary}

The first measurement of coherent and incoherent $\mathrm{J} / \psi$ photoproduction and two-photon production of di-lepton pairs in $\mathrm{Pb}-\mathrm{Pb}$ collisions at the $\mathrm{LHC}$ has been presented and compared with model calculations. The $\mathrm{J} / \psi$ photoproduction cross sections provide a powerful tool to constrain the nuclear gluon shadowing in the region $x \sim 10^{-3}$. The coherent $\mathrm{J} / \psi$ cross section is found to be in good agreement with the model which incorporates the nuclear gluon shadowing according to the EPS09 parameterization (AB-EPS09). Models which include no nuclear gluon shadowing are inconsistent with the measured results, such as those which use the Glauber model to incorporate nuclear effects. The AB-HKN07 and AB-EPS08 models contain too little or too much shadowing, respectively, to match the data. Our results are about 3 sigma higher than the RSZ-LTA model prediction, although a deviation of just 1.5 sigma is found from the model upper limit. Nevertheless the above predictions may have large uncertainties coming not only from the parametrization of the nuclear gluon distribution but also from the selection of the hard scale, the contributions from the higher-order terms and the treatment of the photon fluctuation to a quark-antiquark pair. The current measurement will contribute to resolve these uncertainties. None of the three existing models predicts the incoherent photoproduction cross section correctly, but STARLIGHT predicts a correct incoherent-to-coherent ratio.

\section{References}

[1] E. Fermi, Nuovo Cimento 2 143-158 (1925) 

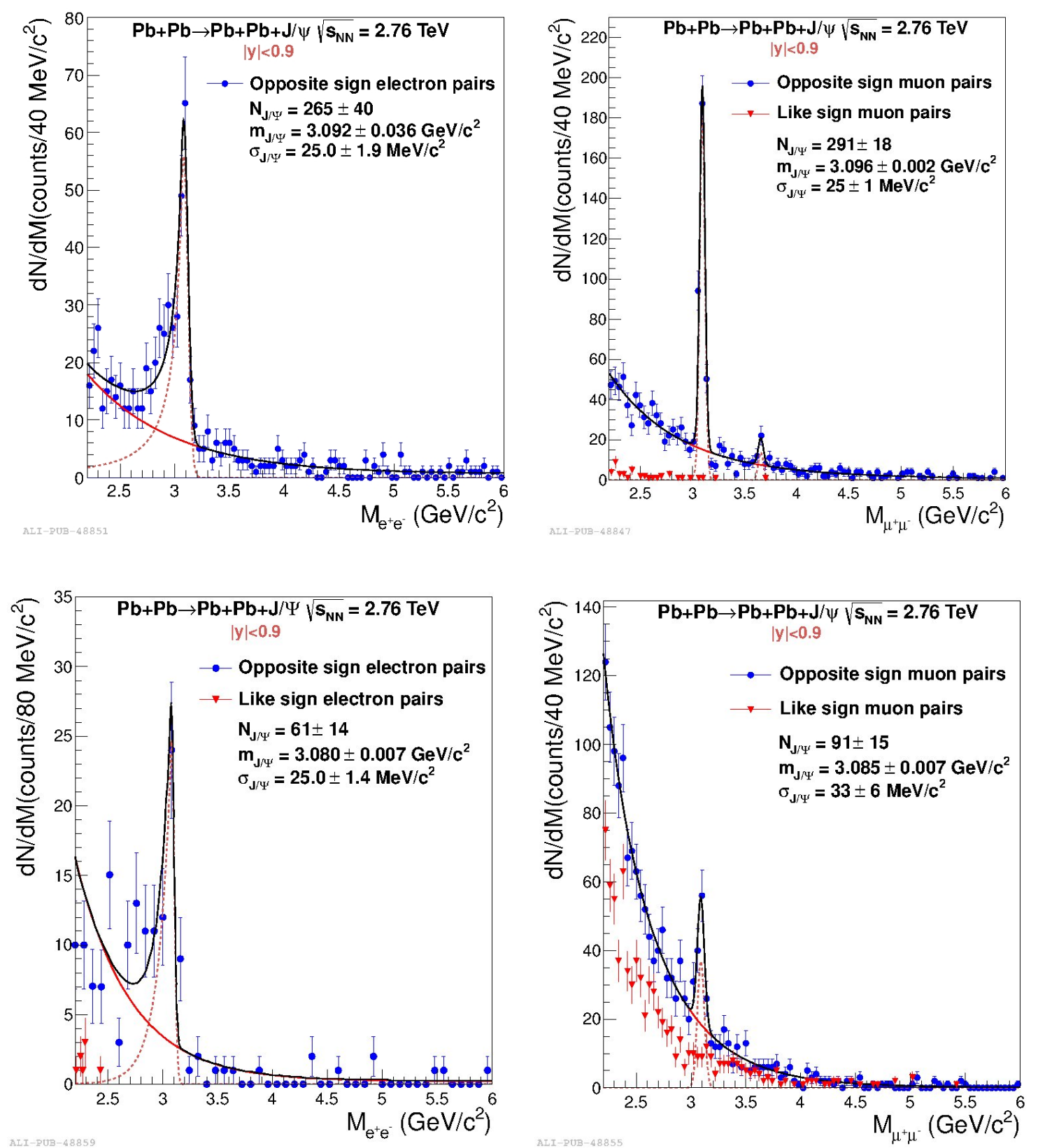

Figure 2: Invariant mass distributions for ultra-peripheral $\mathrm{Pb}-\mathrm{Pb}$ collisions at $\sqrt{\mathrm{s}_{\mathrm{NN}}}=2.76 \mathrm{TeV}$ and $|y|<0.9$ for events satisfying the event selection, in the invariant mass range $2.2<M_{\mathrm{inv}}<6 \mathrm{GeV} / c^{2}$. Coherent enriched sample (top) and incoherent enriched sample (bottom) for electrons (left) and muons (right). The coherent request is fulfilled with transverse momenta below $200 \mathrm{MeV} / c$ (muon channel) or $300 \mathrm{MeV} / c$ (electron channel) and less than 6 neutrons in the ZDC. Blue (red) circles (triangles) are opposite-sign (likesign) pairs. The continuous line is the result of the fit as described in the text.

[2] C. F. Weizsäcker, Z. Phys. 88 612-625 (1934)

[3] E. J. Williams, Phys. Rev. 45 729-730 (1934) 


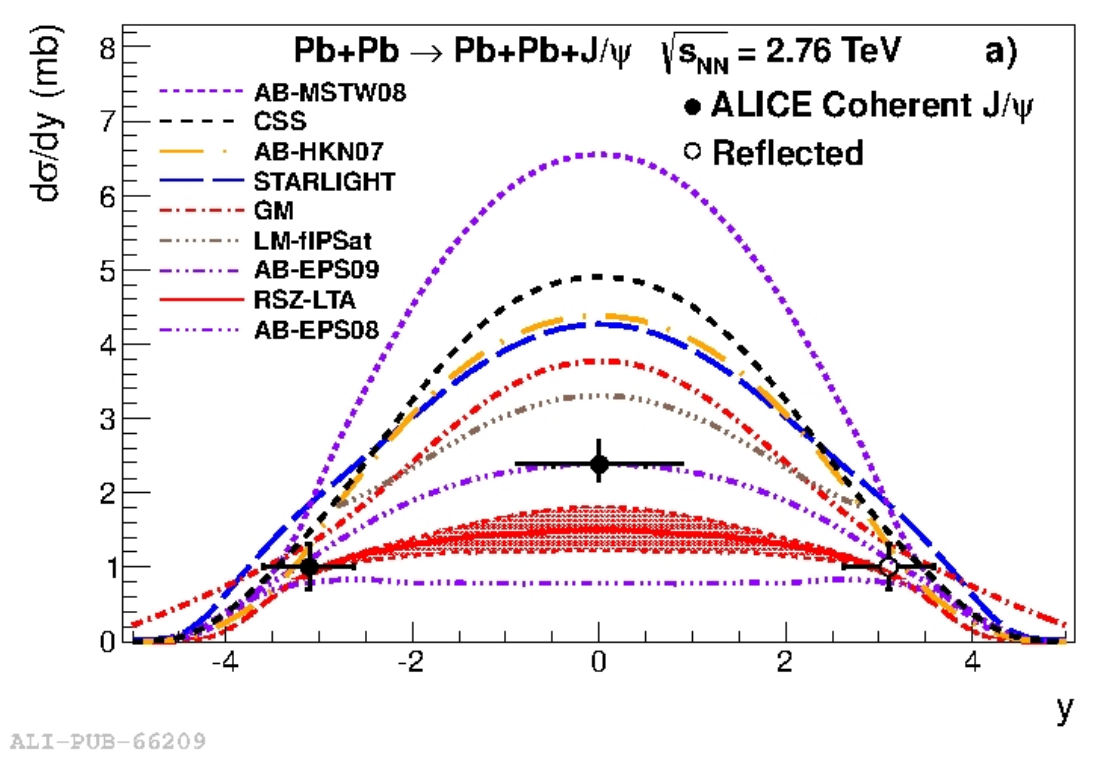

Figure 3: Comparison among the published value of the cross section at forward rapidity [1], the result at central rapidities and several theoretical models. The error is the quadratic sum of the statistical and systematic errors.

[4] A. J. Baltz et al., Phys. Rept. 4581 (2008)

[5] C. A. Bertulani, S. R. Klein, J. Nystrand, Ann. Rev. Nucl. Part. Sci. 55271 (2005)

[6] L. Frankfurt, W. Koepf, M. Strikman, Phys. Rev. D 57512 (1998)

[7] A. D. Martin, C. Nockles, M.G. Ryskin, T. Teubner, Phys. Lett. B 662252 (2008)

[8] V. Rebyakova, M. Strikman, M. Zhalov, Phys. Lett. B 710647 (2012)

[9] K. J. Eskola, H. Paukkunen, C. A. Salgado, JHEP 0904065 (2009)

[10] K. Aamodt et al. [ALICE Collaboration] , JINST 3 S08002 (2008)

[11] B. Abelev et al. [ALICE Collaboration], Phys. Lett. B 718 1273-1283 (2013)

[12] B. Abelev et al. [ALICE Collaboration], Eur. J. Phys. C 73, 2617 (2013)

[13] A. J. Baltz, Phys. Rev. C 80 (2009) 034901.

[14] A. Aktas et al. Eur.Phys. J.C46:585-603,2006

[15] S. Chekanov et al., Nucl. Phys. B695 (2004) 3 